\title{
The accommodation coefficient of the liquid at temperatures below the boiling
}

\author{
Elena E. Bulba \\ National Research Tomsk Polytechnic University, Tomsk 634050, Russia
}

\begin{abstract}
Are carried out experimental investigation of the laws of vaporization at temperatures below the boiling point. Is determined the mass rate of evaporation of distilled water in large intervals of time at different temperatures in order to sound conclusions about the stationarity of the process of evaporation of the liquid in the conditions of the experiments performed, and also studied the effect of temperature on the rate of evaporation. Accommodation coefficient is defined in the mathematical expression of the law of HertzKnudsen for standart substance used in the experiments.
\end{abstract}

\section{Introduction}

Processes vaporization by heating water to a temperature below the boiling point plays an important role in heat-and-power engineering [1], and other industries for extinguishing different fires [2]. But the choice of technologies to suppress fires recently conducted empirically due to the lack of constant characterizing the process of evaporation at moderate temperatures [3] (e.g., accommodation coefficient in the mathematical expression of the law of Hertz-Knudsen). Recently performed experimental studies of heat and mass transfer in heavy water droplets have evaporated when driving through the flames. But such results is not enough to establish the empirical constants evaporation.

The aim of the present work is the experimental study of the laws of vaporization at temperatures below the boiling point.

\section{Experimental procedure}

Experiments were carried out using a heating chamber, which was placed in a vessel with a specific area of evaporation, the mass of which is measured by a laboratory balance. The vessel was filled with distilled water $150 \mathrm{ml}$. Electrocontact thermometer to monitoring the temperature in the heat chamber. Measures the atmospheric pressure and humidity at which conducted experimental studies. Multimeter set to "temperature measurement", thermocouple was placed in the test liquid, and included a heat chamber. Upon reaching the temperature of the liquid in the vessel equal to the temperature in the heating chamber, the vessel was removed from the water and the measured weight. The vessel was then placed in a fluid drier, and the experiment was conducted. At the end of a specified period of time the

This is an Open Access article distributed under the terms of the Creative Commons Attribution License 4.0, which permits unrestricted use, distribution, and reproduction in any medium, provided the original work is properly cited. 


\section{EPJ Web of Conferences}

Table 1. The dependence of the rate of evaporation of distilled water on the time.

\begin{tabular}{|c|c|c|c|c|c|}
\hline $\mathrm{T}, \mathrm{K}$ & \multicolumn{5}{|c|}{313} \\
\hline $\mathrm{t}, \mathrm{c}$ & 1500 & 3000 & 4500 & 6000 & 7500 \\
\hline $\mathrm{w} \cdot 10^{3}, \mathrm{~kg} /\left(\mathrm{m}^{2} \mathrm{c}\right)$ & 0.11 & 0.112 & 0.11 & 0.11 & 0.11 \\
\hline $\mathrm{T}, \mathrm{K}$ & \multicolumn{5}{|c|}{353} \\
\hline $\mathrm{t}, \mathrm{c}$ & 1500 & 3000 & 4500 & 6000 & 7500 \\
\hline $\mathrm{w} \cdot 10^{3}, \mathrm{~kg} /\left(\mathrm{m}^{2} \mathrm{c}\right)$ & 1.29 & 1.29 & 1.291 & 1.29 & 1.291 \\
\hline $\mathrm{T}, \mathrm{K}$ & \multicolumn{5}{|c|}{363} \\
\hline $\mathrm{t}, \mathrm{c}$ & 1500 & 3000 & 4500 & 6000 & 7500 \\
\hline $\mathrm{w} \cdot 10^{3}, \mathrm{~kg} /\left(\mathrm{m}^{2} \mathrm{c}\right)$ & 2.01 & 2.1 & 2.1 & 2.0 & 2.2 \\
\hline
\end{tabular}

vessel was weighed and the amount of evaporated liquid. The experiment was repeated at least 3 times for a constant temperature chamber at a specified interval of time.

Heating is ensured by wire heater located on the heating coil. Such heating panels have good heat transfer and provide uniform heating of the surface of the working chamber. Comparing natural ventilation heat chamber.

In the lower part there are air ducts for supply of cold air. Ventilation rate was adjusted lower ventilation flap. In the upper portion of the working chamber located holes temperature measuring device and to reset moisture vapor. The basic quantities characterizing the evaporation process, the speed of the phase transition were, the surface area of the liquid, which evaporates, and the temperature. The first characteristic determined by the surface density of the steam flow supplied per unit time into the gas phase per unit surface area of the liquid.

Investigations of mass evaporation rate of distilled water in large intervals of time at different temperatures in order to sound conclusions about the stationarity of the process of evaporation of the liquid in the conditions of the experiments performed. Selecting an object of research for several reasons. Firstly, in preliminary experiments carried out it was found quite significantly (from 3 to $7 \%$ ), the deviation of evaporation rates of the three kinds of typical water obtained from a variety of sources. Rather, it is a consequence of the influence of mineral content on the phase transformation at the "liquidvapor". Mineral components (e.g., salts) changing the configuration of the interface and the surface tension forces. Changes accordingly and the accommodation coefficient characterizing the number of molecules of steam coming off the surface evaporation. Distilled water was selected as the specific standard for the analysis of the results of subsequent studies.

\section{Results and discussion}

By analyzing the results (Table 1), it can be concluded that the mass evaporation rate of the distilled water with the passage of time does not change throughout the temperature range studied (evaporation process in this case is stationary).

Here, $\mathrm{T}$ - temperature in a heat chamber, $\mathrm{t}$ - duration of the experiment, $\mathrm{w}(\mathrm{kg} /(\mathrm{s} \cdot \mathrm{m} 2)$ - mass rate of evaporation of the liquid.

At the next stage of the experiment the dependence of the mass rate of evaporation temperature.

Figure 1 shows the experimental results when heating in the range of $313 \mathrm{~K}$ to $363 \mathrm{~K}$ ata rate depending on the phase transition temperature. Confidence intervals are given describing error measurements.

Processing of the results of experiments carried out using well established techniques [4]. For each value of the temperature at a given interval of time $(t=20 \mathrm{~min})$. Was carried out from three to five measurements. Depending on the experimental W $(\mathrm{T})$ is calculated accommodation coefficient, which depends on the nature and state of the surface, as well as the properties of the vapor and the temperature.

From the well-known formula for finding the mass evaporation rate expression is derived for the calculation of the accommodation coefficient (Hertz-Knudsen law): 


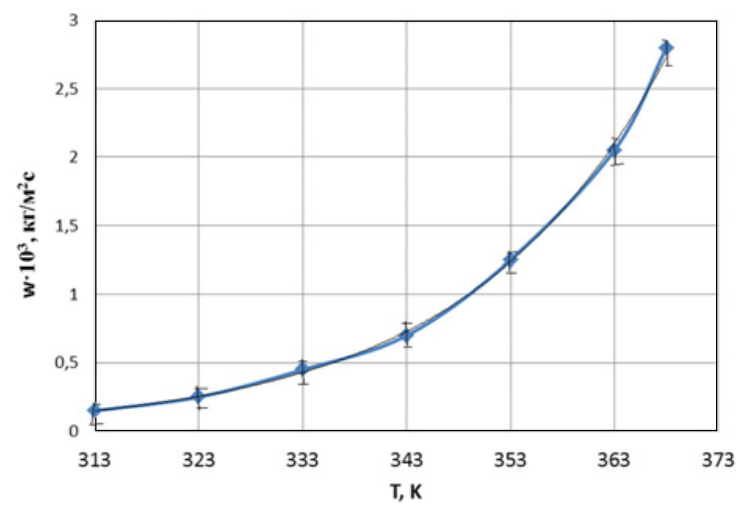

Figure 1. The dependence of the mass evaporation rate of distilled water vs. temperature.

Table 2. Coefficient of accommodation distilled water.

\begin{tabular}{|l|l|l|l|l|}
\hline $\mathrm{T}, \mathrm{K}$ & $W_{i c p} \cdot 10^{3}, \mathrm{~kg} /\left(\mathrm{m}^{2} \cdot \mathrm{c}\right)$ & $P^{\mathrm{H}}, \mathrm{Pa}$ & $P^{*}, \mathrm{~Pa}$ & The accommodation coefficient, $\mathrm{A} \cdot 10^{3}$ \\
\hline 313 & 0.11 & 7375 & 6416 & 0.13 \\
\hline 323 & 0.2 & 12330 & 10727 & 0.15 \\
\hline 333 & 0.43 & 19920 & 17330 & 0.17 \\
\hline 343 & 0.7 & 311160 & 27109.2 & 0.21 \\
\hline 353 & 1.29 & 47360 & 41203.2 & 0.214 \\
\hline 363 & 2.1 & 70110 & 60995.7 & 0.236 \\
\hline
\end{tabular}

where $\mathrm{W}$ - mass evaporation rate, $\mathrm{kg} / \mathrm{m} 2 \cdot \mathrm{s} ; \mathrm{PH}$ - vapor pressure, $\mathrm{Pa} ; \mathrm{P}^{*}$ - partial pressure of the evaporating component calculated at a relative humidity $\varphi=0,87, \mathrm{PA} ; \mathrm{R}=8,31$ - universal gas constant, $\mathrm{J} / \mathrm{mol} \cdot \mathrm{K} ; \mathrm{M}$ - molecular weight; $\mathrm{T}$ - temperature of the substance, $\mathrm{K} ; \mathrm{A}$ - accommodation coefficient.

Table 2 shows the calculated values of the accommodation coefficient in the temperature range from $313 \mathrm{~K}$ to $363 \mathrm{~K}$ for the distilled water.

Analyzing the results of Table 2, it can be noted that with increasing temperature of the liquid increases and the accommodation coefficient.

\section{Conclusion}

Obtained in the experiments carried out, the results are not only empirical basis for mathematical modeling of heat and mass transfer processes in heavy water droplets have evaporated when driving through high-temperature gases [2]. Experimental dependences of the mass rate of evaporation of water to investigate the temperature are objective prerequisites for the determination of the accommodation coefficient in the mathematical expression of the law of Hertz-Knudsen. Knowledge of the numerical value of this quantity allows for conducting similar experiments with other types of water to analyze the influence of various mineral additives on the phase transformation. In the future, you can use the considered experimental data for the development of mathematical models of intensive evaporation of flammable liquids in a local heating [5]. 


\section{References}

[1] Kuznetsov G.V., Al-Ani, Sheremet M.A. Numerical analysis of convective heat transfer in a closed two-phase thermosyphon// Journal of Engineering Thermophysics. - 2011. - P. 201-210

[2] Vysokomornaya O.V., Kuznetsov G.V., Strizhak P.A. Heat and mass transfer in the process of movement of water drops in a hightemperature gas medium// Journal of Engineering Physics and Thermophysics. - 2013, - P. 1-7

[3] Kuznetsov G.V., Strizhak P.A. 3D problem of heat and mass transfer at the ignition of a combustible liquid by a heated metal particle//Journal of Engineering thermophysics. - 2009. - 18. No 1. C. $72-79$

[4] Arkhipov V.A., Berezikov A.P. Basic theory of engineering and physical experiment. - Tomsk: Tomsk Polytechnic University Publishing House, 2008.- P. 206

[5] Kuznetsov G.V., Strizhak P.A. The influence of heat transfer conditions at the hot particle-liquid fuel interface on the ignition characteristics// Journal of Engineering Thermophysics. 2009. 18. No 2. C. $162-167$ 